\title{
Using the method of integral Fourier transform to solve the problem of nonstationary deformation of an elastic medium
}

\author{
Volodymyr Sapehin ${ }^{1, *}$ \\ ${ }^{1}$ Institute of Geotechnical Mechanics named by N. Poljakov of National Academy of Sciences of \\ Ukraine, 49005, Dnipro, Simferopolska Str., 2a, Ukraine
}

\begin{abstract}
For the first time, for solving the wave equation, the method of the integral Fourier transform for an elastic medium under the action of variable internal pressure was used. This equation contains singularities of the second kind, which were excluded from the calculation by the half division method. The main parameters of the process of non-stationary deformation affecting the magnitude of stresses and displacements for the case of plane deformation are established.
\end{abstract}

\section{Introduction}

Gas-bearing coal and sandstone are a complex fractured-porous medium with different systems of macropores, micropores and cracks of various specific lengths, thicknesses, medium gaping and specific surfaces. The impact on the well is carried out in a limited volume of the gas-bearing medium under conditions when the mountain massif is in a loaded state and when there is no intensive gas filtration and active crack growth. In this case, in the first approximation, a defective stochastic medium can be considered, according to the hypothesis of R.L. Salganik, as homogeneous, and the dynamic problem of determining stresses and displacements can be solved by the elastic homogeneous medium model [1]. This task has a wide practical application in the mining industry. After the internal pressure of the working agent is quickly released in the well, tensile stresses occur in the marginal zone of the cylindrical cavity of the well, thereby stimulating the formation of cracks and gas release from the array. In this case, the effect of inertial forces is used. Many analytical solutions are obtained mainly for simple cases, namely, for a homogeneous elastic or elastoplastic model of the medium. The most famous decision on the dynamic loading of an elastic thick-walled hollow cylinder was obtained by A.N. Krylov by the method of separation of Fourier variables in the form of an infinite series in its own forms of oscillations.

It is widely used in the calculation of dynamic stresses and displacement around wells under the action of a variable internal load [2].

The solution of the wave equation in displacements is a discontinuous function, has a limited region of convergence due to the fact that an infinite series with features of the second kind is used. In this case, the known method cannot be applied to calculate dynamic

*Corresponding author: vladimirsapegin10@gmail.com 
stresses around wells with large external boundaries. The area of this solution is limited by the ratios of the inner and outer radii of the elastic cylinder not exceeding 1 in 10 [2].

\section{Problem definition and solution method}

Solving the problems of the theory of elasticity for infinite and semi-infinite bodies is effectively performed by the method of the integral Fourier transform [3]. Using the integral Fourier transform, we consider a plane axisymmetric deformation of an elastic medium for a well that has been drilled through a rock plug either across the strike or along the strike of the coal seam. Arbitrarily varying temporal pressure was applied inside the well $\psi(t)$, and at infinity the rock pressure was assumed to be zero.

The initial static stress field or rock pressure forces can be taken into account by the well-known Lame equations for a hollow thick-walled cylinder and the KolosovMuskhelishvili solution, which takes into account the different components of the external static stress field for the case of plane strain.

The resulting stress value in the vicinity of the well will be determined taking into account the static (rock pressure) and dynamic components of the stress fields from the action of the internal pressure using the linear superposition method.

The displacement vector will have a radial component depending on the radius and time. We solve the problem in a cylindrical coordinate system. The basic wave equation for the motion of an elastic medium under the influence of variable internal pressure will have the form [2]:

$$
r^{2} \cdot \frac{\partial^{2} u_{r}}{\partial r^{2}}+r \cdot \frac{\partial u_{r}}{\partial r}-u_{r}=\frac{r^{2}}{v_{p}^{2}} \cdot \frac{\partial^{2} u_{r}}{\partial t^{2}},
$$

where $u_{r}(r, t)$ - radial displacement of the elastic medium, $\mathrm{m} ; t$ - the time of the deformation process, $\mathrm{s} ; \mathrm{v}_{p}$ - the velocity of the elastic wave, $\mathrm{m} / \mathrm{s}$.

Boundary conditions on the inner and outer surface of the well:

$$
\left.\sigma_{r}\right|_{r=r_{0}}=-\psi(t) ;\left.\quad \sigma_{r}\right|_{r=r_{N}}=0,
$$

where $r_{0}$ - the inner radius of the well, $\mathrm{m} ; r_{N}$ - outer radius of the cylinder, $\mathrm{m}$.

Applying the direct Fourier transform with respect to time $t$ to equation (1) and boundary conditions (2), we obtain an ordinary second-order differential equation for the displacement transform and the transformation parameter $\omega$. This equation is the Bessel equation (3), the general solution of which is known $(4,5)[3]$ :

$$
\begin{gathered}
r^{2} \cdot \frac{d^{2} u_{0 r}}{d r^{2}}+r \cdot \frac{d u_{0 r}}{r}+\left(\frac{r^{2}}{v_{p}^{2}} \cdot \omega^{2}-1\right) \cdot u_{0 r}=0, \\
\left.\sigma_{0 r}\right|_{r=r_{0}}=-\bar{\psi}(\omega) ;\left.\quad \sigma_{0 r}\right|_{r=r_{N}}=0 ; \\
u_{0 r}=A(\omega) \cdot J_{1}\left(\frac{\omega \cdot r}{v_{p}}\right)+B(\omega) \cdot Y_{1}\left(\frac{\omega \cdot r}{v_{p}}\right),
\end{gathered}
$$

where $A(\omega), B(\omega)$ - arbitrary constants that depend on the transformation parameter $\omega$ and are determined from the boundary conditions (4); $\omega$ - transformation parameter, $1 / \mathrm{s} ; J_{1}$, $Y_{1}$ - Bessel functions of the first and second kind of the first order; $u_{0_{r}}$ - transformant displacement, $\mathrm{m} ; \bar{\psi}(\omega)$ - load transformant; $\sigma_{0_{r}}$ - transformant of radial stress, Pa. 
Using the known formulas for the case of plane strain for radial and tangential stresses in displacements and applying to them the direct integral Fourier transform with respect to time, we obtain:

$$
\sigma_{0 r}=\frac{E}{1-v^{2}} \cdot\left(\frac{d u_{0 r}}{d r}+v \cdot \frac{u_{0 r}}{r}\right) ; \quad \sigma_{0 \theta}=\frac{E}{1-v^{2}} \cdot\left(\frac{u_{0 r}}{r}+v \cdot \frac{d u_{0 r}}{r}\right),
$$

where $E$ - is the modulus of elasticity, Pa; $v$ - Poisson's ratio; $\sigma_{0_{r}}$ - transformant radial stress, $\mathrm{Pa} ; \sigma_{0 \theta}$ - transformant tangential stress, $\mathrm{Pa}$.

Substituting the expression for bias conversion (5) into equations (6) and using formulas for differentiating Bessel functions of the first kind and second kind and formulas connecting the Bessel functions of the first and second kind of zero and first orders, we obtain for the transformant radial and tangential stress with the expression (6) and the notation $\xi=\omega \cdot r_{0} / v_{p}$ the following formulas (7) and (8):

$$
\begin{aligned}
& \frac{\sigma_{0 r}}{2 G}=A(\xi) \cdot\left(\frac{1}{1-v} \cdot \frac{\xi}{r_{0}} \cdot J_{0}\left(\xi \cdot \frac{r}{r_{0}}\right)-\frac{1}{r} \cdot J_{1}\left(\xi \cdot \frac{r}{r_{0}}\right)\right)+ \\
& +B(\xi) \cdot\left(\frac{1}{1-v} \cdot \frac{\xi}{r_{0}} \cdot Y_{0}\left(\xi \cdot \frac{r}{r_{0}}\right)-\frac{1}{r} \cdot Y_{1}\left(\xi \cdot \frac{r}{r_{0}}\right)\right), \\
& \frac{\sigma_{0 \theta}}{2 G}=A(\xi) \cdot\left(\frac{v}{1-v} \cdot \frac{\xi}{r_{0}} \cdot J_{0}\left(\xi \cdot \frac{r}{r_{0}}\right)+\frac{1}{r} \cdot J_{1}\left(\xi \cdot \frac{r}{r_{0}}\right)\right)+ \\
& +B(\xi) \cdot\left(\frac{v}{1-v} \cdot \frac{\xi}{r_{0}} \cdot Y_{0}\left(\xi \cdot \frac{r}{r_{0}}\right)+\frac{1}{r} \cdot Y_{1}\left(\xi \cdot \frac{r}{r_{0}}\right)\right),
\end{aligned}
$$

where $J_{0}, Y_{0}$ - are Bessel functions of the first and second kind of order zero; $G$ - is the shear modulus, $\mathrm{Pa}$.

To simplify the writing of formulas, we introduce the notation and function:

$$
\begin{gathered}
\xi_{N}=r_{N} / r_{0} \cdot \xi ; \beta=r_{N} / r_{0} ; \\
Y_{j n}\left(\xi_{N}, \xi\right)=J_{j}\left(\xi_{N}\right) \cdot Y_{n}(\xi)-J_{n}(\xi) \cdot Y_{j}\left(\xi_{N}\right) ; \quad r_{1}=r / r_{0} ; q=(-1+v) .
\end{gathered}
$$

Formulas for defining arbitrary constants will have the following form (9), (10):

$$
\begin{aligned}
& A(\xi)=\frac{-r_{0} \cdot q \cdot \bar{\psi}(\xi) \cdot v_{p} \cdot\left(\beta \cdot v_{p} \cdot \xi \cdot Y_{0}(\beta \cdot \xi)+q \cdot Y_{1}(\beta \cdot \xi) \cdot v_{p}\right)}{C}, \\
& B(\xi)=\frac{r_{0} \cdot q \cdot \bar{\psi}(\xi) \cdot v_{p} \cdot\left(\beta \cdot v_{p} \cdot \xi \cdot J_{0}(\beta \cdot \xi)+q \cdot J_{1}(\beta \cdot \xi) \cdot v_{p}\right)}{C},
\end{aligned}
$$

where $C=2 G \cdot\left[r_{0} \cdot r_{N} \cdot\left(v_{p} / r_{0} \cdot \xi\right)^{2} \cdot Y_{00}(\beta \cdot \xi, \xi)+q \cdot v_{p} \cdot\left(v_{p} / r_{0} \cdot \xi \cdot\left(r_{0} \cdot Y_{10}(\beta \cdot \xi, \xi)+\right.\right.\right.$

$$
\left.\left.+r_{N} \cdot Y_{01}(\beta \cdot \xi, \xi)\right)+q \cdot Y_{11}(\beta \cdot \xi, \xi) \cdot v_{p}\right) \text {, }
$$

$\bar{\psi}(\xi)$ - load transformant.

Consider the most common case in the mountain practice of calculating displacements and stresses in the vicinity of a well for pressure, which increases according to a linear law, in a regime close to static. In this feed mode, the maximum accumulation of energy in the 
elastic coal-rock array occurs, and then it is quickly reset, for example, according to a linear law.

The calculated scheme of the pressure change of the working agent in time in the well is shown in Figure 1 and formula (13):

$$
\psi(t)=\left\{\begin{array}{l}
k_{1} \cdot t, \quad 0 \leq t \leq t_{1} \\
-k_{2} \cdot\left(t-t_{2}\right), \quad t_{1} \leq t \leq t_{2}, \\
0, \quad t \geq t_{2}
\end{array}\right.
$$

where $k_{1}=P_{0} / t_{1}, \mathrm{~Pa} / \mathrm{s} ; k_{2}=P_{0} /\left(t_{2}-t_{1}\right), \mathrm{Pa} / \mathrm{s} ; P_{0}$ - maximum value (amplitude) of internal pressure, $\mathrm{Pa} ; t_{1}$ - is the total time of pressure rise, counted from the origin, $\mathrm{s} ; t_{2}$ - is the time from the origin to the moment of complete pressure relief, $\mathrm{s} ;\left(t_{2}-t_{1}\right)$ - time of pressure relief $t_{c}$, s.

Let us write the transformant from the load acting on different loading sites using the known sine-integral Fourier transform using the formula (14):

$$
\bar{\psi}(\xi)=\sqrt{\frac{2}{\pi}} \cdot \frac{P_{0} \cdot r_{0}{ }^{2}}{\left(t_{1}-t_{2}\right) \cdot v_{p}{ }^{2}} \cdot \frac{\left(-t_{2} \cdot \sin \left(\frac{t_{1} \cdot v_{p} \cdot \xi}{r_{0}}\right)+t_{1} \cdot \sin \left(\frac{t_{2} \cdot v_{p} \cdot \xi}{r_{0}}\right)\right)}{t_{1} \cdot \xi^{2}},
$$

To obtain true displacements and stresses, the inverse integral Fourier transform is performed using formulas (15-17)

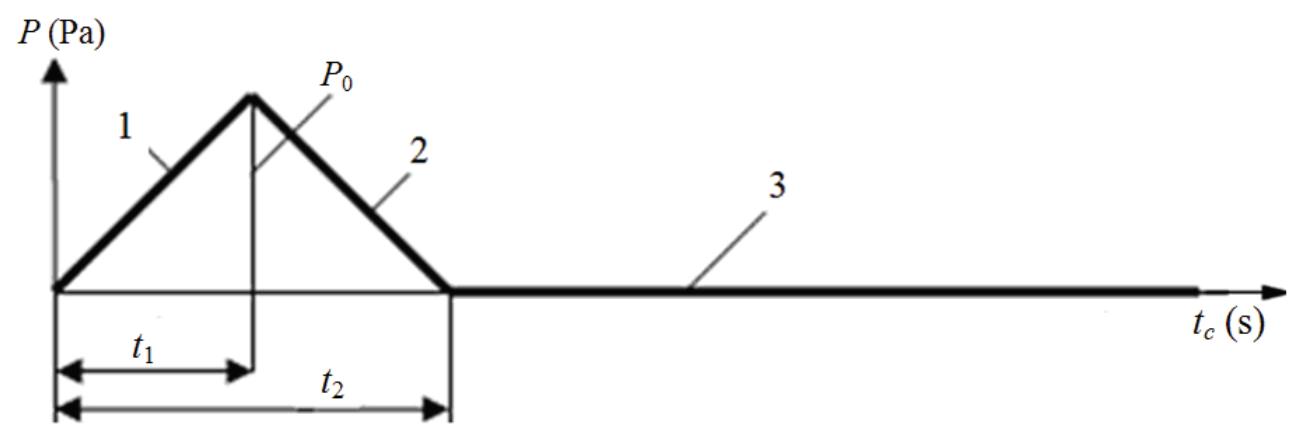

Fig. 1. Scheme of pressure change $P$ of the working agent from time $t$ in the well at various stages of loading: 1 - pressure feed $0 \leq t \leq t_{1} ; 2$ - pressure relief $t_{1} \leq t \leq t_{2} ; 3$ - the action of inertial forces $t \geq t_{2}$; $t_{2}-t_{1}=t_{c}$ - time of pressure relief from the well; $P_{0}$ - the maximum value of the working agent pressure before the pressure is released.

$$
\begin{aligned}
& u_{r}=\frac{2}{\pi} \cdot \frac{v_{p}}{r_{0}} \cdot \int_{0}^{\infty} u_{0 r}\left(\xi, r / r_{0}\right) \cdot \sin (\xi \cdot d) d \xi, \\
& \sigma_{r}(r, t)=\frac{2}{\pi} \cdot \frac{v_{p}}{r_{0}} \cdot \int_{0}^{\infty} \sigma_{0 r}\left(\xi, r / r_{0}\right) \cdot \sin (\xi \cdot d) \cdot d \xi \\
& \sigma_{\theta}(r, t)=\frac{2}{\pi} \cdot \frac{v_{p}}{r_{0}} \cdot \int_{0}^{\infty} \sigma_{0 \theta}\left(\xi, r / r_{0}\right) \cdot \sin (\xi \cdot d) \cdot d \xi
\end{aligned}
$$

In the dimensionless form of displacement and stress, taking into account the notation (9) and formulas (14-17), are determined by the formulas: 


$$
\begin{gathered}
u_{r} / r_{0}=\left(2 \cdot v_{p} / \pi \cdot r_{0}\right) \cdot \int_{0}^{\infty}\left\{( 1 / G \cdot Y _ { 1 0 } ( \xi , \xi ) ) \cdot \left[\bar{A} \cdot\left(Y_{10}\left(\xi \cdot r_{1}, \xi\right)-(1 / k \cdot \xi) \cdot Y_{11}\left(\xi \cdot r_{1}, \xi\right)\right)-\right.\right. \\
\left.\left.-(1 / k \cdot \xi) \cdot Y_{11}\left(\xi \cdot r_{1}, \xi\right)\right] \cdot \bar{\psi}(\xi)\right\} \cdot \sin [d \cdot \xi] d \xi \\
\frac{\sigma_{r}}{P_{0}}=\left(2 v_{p} / \pi \cdot r_{0}\right) \cdot \int_{0}^{\infty}\left\{( 1 / Y _ { 1 0 } ( \xi , \xi ) ) \cdot \left[\overline { A } \cdot \left(k \cdot \xi \cdot Y_{00}\left(\xi \cdot r_{1}, \xi\right)-Y_{01}\left(\xi \cdot r_{1}, \xi\right)-r_{1} \cdot Y_{10}\left(\xi \cdot r_{1}, \xi\right)+\right.\right.\right. \\
\left.\left.\left.+\left(1 / k \cdot \xi \cdot r_{1}\right) \cdot Y_{11}\left(\xi \cdot r_{1}, \xi\right)\right)+\left(1 / k \cdot \xi \cdot r_{1}\right) \cdot Y_{11}\left(\xi \cdot r_{1}, \xi\right)-Y_{01}\left(\xi \cdot r_{1}, \xi\right)\right] \cdot \bar{\psi}(\xi)\right\} \cdot \sin [d \cdot \xi] d \xi, \\
\quad \frac{\sigma_{\theta}}{P_{0}}=\left(2 \cdot v_{p} / \pi \cdot r_{0}\right) \int_{0}^{\infty}\left\{( 1 / k \cdot \xi \cdot Y _ { 1 0 } ( \xi , \xi ) ) \cdot \left[\overline { A } \cdot \left(\left(k \cdot v \cdot \xi^{2} / 1-2 v\right) \cdot Y_{00}\left(\xi \cdot r_{1}, \xi\right)+\right.\right.\right. \\
\left.\quad+\left(k \cdot \xi / r_{1}\right) \cdot Y_{10}\left(\xi \cdot r_{1}, \xi\right)-(v \cdot \xi / 1-2 v) \cdot Y_{01}\left(\xi \cdot r_{1}, \xi\right)-\left(1 / r_{1}\right) \cdot Y_{11}\left(\xi \cdot r_{1}, \xi\right)\right)- \\
\left.\left.\quad-\left((v \cdot \xi / 1-2 v) \cdot Y_{01}\left(\xi \cdot r_{1}, \xi\right)+\left(1 / r_{1}\right) \cdot Y_{11}\left(\xi \cdot r_{1}, \xi\right)\right)\right] \cdot \bar{\psi}(\xi)\right\} \cdot \sin [d \cdot \xi] \cdot d \xi, \\
Y_{01}(\xi \cdot \beta, \xi)-\frac{1}{k \cdot \xi \cdot \beta} \cdot Y_{11}(\xi \cdot \beta, \xi)
\end{gathered}
$$

where $d=v_{\mathrm{p}} \cdot t / r_{0}$ is the dimensionless parameter (dimensionless time) of the process;

$k=1 /(v-1)-$ dimensionless coefficient.

\section{Results and discussion}

The initial data for the calculation: $v_{p}=1000 \mathrm{~m} / \mathrm{s}, t_{c}=0.001 \mathrm{~s}, r=r_{0}=0.1 \mathrm{~m}$.

The calculation was made according to the formulas (18-21) in the following order: a dimensionless process parameter was specified, the elastic wave velocity $v_{p}$, the coefficient Poisson's ratio $v$, shear modulus $G$, cavity radius $r_{0}$, the value of the current medium radius $r$, the amplitude of the load $P_{0}$ and the law of its variation with time $\psi(t)$.

The calculation of the Fourier - Bessel integrals of rapidly oscillating functions was carried out according to a standard program using the Gauss - Kronrod quadrature formula [4] taking into account the periodic system of singularities of infinite discontinuity. They were first isolated from equation (21), and then excluded from the calculation before integration.

Based on the calculations carried out using formulas (18-20), it was found that the nature of the change in radial (tangential) stresses over time after the cessation of the force is oscillatory, with the same period of damped oscillations. The elastic system gradually returns to a stable initial state, as the action of inertia forces decreases, with alternating changes in tensile and compressive stresses of opposite sign.

It is established that the smaller the pressure release time, the greater the tensile stresses on the inner contour of the well cavity, which differ in hyperbolic dependence.

For given parameters of an elastic medium, when the reset time $t_{c}$ is less than $0.01 \mathrm{~s}$, a sharp increase in radial and tangential stresses is observed. This is due to the high rate of drop in the internal pressure of the working agent in the well.

Figure 2 shows the dependence of the radial tensile stresses on the reset time $t_{c}$. The initial data for the calculation: $v_{p}=1200 \mathrm{~m} / \mathrm{s}, r_{0}=0.1 \mathrm{~m}$.

Figure 3 shows the dependence of dimensionless tensile stresses on the velocity of the elastic wave at $t_{c}=0.001 \mathrm{~s} ; r_{0}=0.1 \mathrm{~m} ; r_{N}=100 \mathrm{~m}$. 
With an increase in the inner radius of the cylindrical cavity in the studied range from $0.05 \mathrm{~m}$ to $0.3 \mathrm{~m}$, the radial stresses after a discharge of the internal pressure increase in a linear relationship. Radial tensile stresses are directly proportional to the amplitude of the internal load (pressure) before it is released from the well.

The elastic wave velocity is a parameter that relates the deformation and elastic characteristics of the array, depending on the elastic modulus, Poisson's ratio and density of the elastic medium. The greater the elastic modulus and the stronger the medium, or the higher the velocity of the elastic wave, the lower the tensile stresses arising from the discharge of the internal load.

It should be noted that the larger the external radius of the elastic medium, the more reliable the result of calculating displacements and stresses. In this case, the influence of the reflected stress wave from the inner contour of the well decreases and the stresses from the incident wave are determined more accurately.

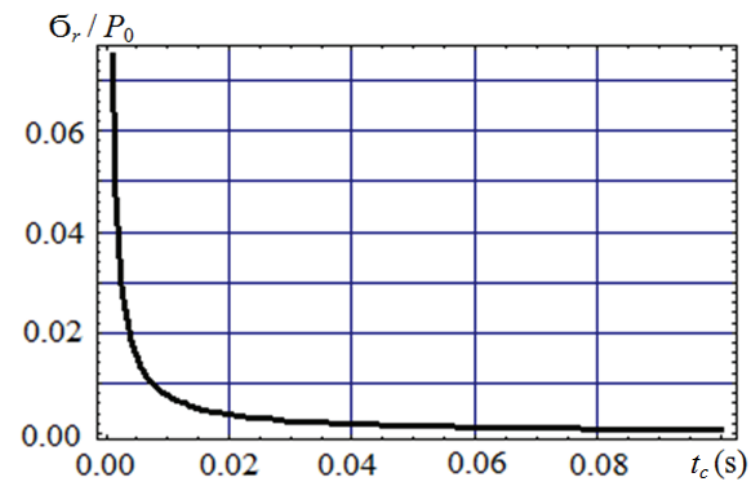

Fig. 2. Change of dimensionless radial tensile stresses $\sigma_{r} / P_{0}$ from the time of pressure drop $t_{c}$.

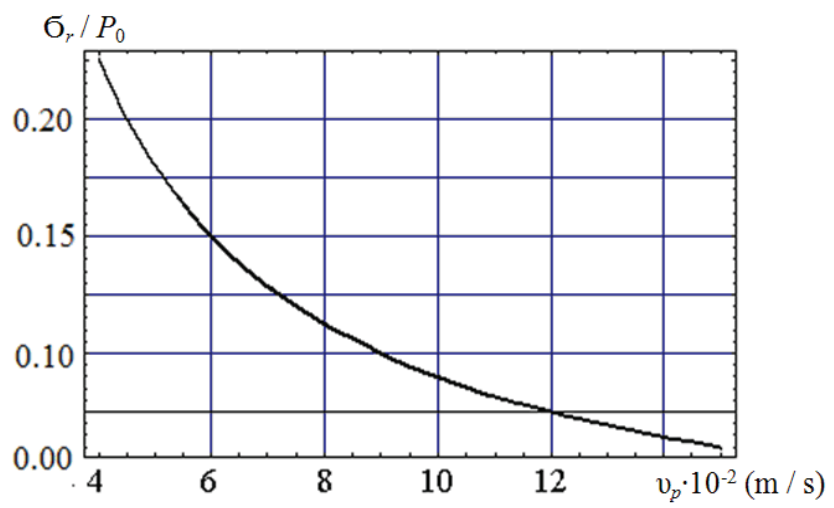

Fig. 3. Change of dimensionless radial compressive stresses $\sigma_{r} / P_{0}$ from the elastic wave velocity $v_{p}$.

\section{Conclusions}

The determining parameters of the dynamic process of non-stationary deformation, affecting the magnitude of the formation of the zone of tensile stresses when the pressure is released from wells, are established. The main factors affecting the deformation process are: the amount of pressure before it is discharged from the well, the diameter of the well, the speed of the elastic wave in the array and the time of pressure drop in the working agent. 
For the first time, a mathematical model of unsteady array deformation in the nearwellbore zone was developed, in which the well-known wave equation was solved by the method of the integral Fourier transform. An analytical solution is obtained for a plane problem for calculating displacements and stresses, which makes it possible to calculate them in semi-infinite and infinite elastic coal-bearing media.

The resulting solution is stable for the ratios of the external radius of the well to the internal equal $\infty \geq r_{N} / r_{0} \geq 1.1$ and, as is typical for the method of integral Fourier transform, converges slowly, which is not very convenient for carrying out operational calculations.

\section{References}

1. Salganik, R.L. (1980). Ob effektivnykh kharakteristikakh materiala s bol'shim chislom treshchin. Vozmozhnost' geofizicheskogo opredeleniya parametrov treshchinovatosti plasta v svyazi s zadachey obespecheniya vybrosoopasnosti. - (Prepr. / IPM AN SSSR, №154), Moskva

2. Krylov, A.N. (1950). O nekotorykh differentsial'nykh uravneniyakh matematicheskoy fiziki, imeyushchikh prilozheniye $\mathrm{v}$ tekhnicheskikh voprosakh: ucheb. posobiye dlya vuzov, Leningrad: Akademiya nauk SSSR

3. Sapehin, V.N. (2011). Nestatsionarnoye deformirovaniye uprugoy sredy pod deystviyem peremennoy vnutrenney nagruzki. Problemy vychislitel'noy mekhaniki $i$ prochnosti konstruktsiy: zb. nauk. prats' / Dnípropetrovs'kiy natsíonal'niy uníversitet. Dnípropetrovs'k: Lira, 17, 239-245

4. Kakhaner, D., Mouler, K. (2001). Chislennyye metody i programmnoye obespecheniye, Moskva: Mir 